\title{
Fractal Growth of Platinum Electrodeposits Revealed by in situ Electron Microscopy
}

Lifen Wang, Jianguo Wen, Huaping Sheng, Dean J. Miller

Center for Nanoscale Materials, Nanoscience and Technology Division, Argonne National Laboratory, Argonne, IL 60439, USA

The fractal growth at solid-liquid interface in electrochemical deposition has long been of interest for physicists, chemists, biologists, and mathematicians [1]. However, the liquid environment poses significant challenges to studies of in situ growth processes. Recent developments for in situ liquid cell transmission electron microscopy provide new opportunities to study electrodeposition growth processes in realistic environments with much better temporal and spatial resolution than ever before, and enables the present experimental research of metal deposition at an electrochemical interface [2].

Here we report an in situ electron microscopy study of dynamic fractal growth of platinum in a miniaturized electrochemical cell. The set of in situ real-time TEM images shown in figure 1 show the early stage evolution of Pt nano-electrodeposition under the potentiostatic mode when constant voltage of $-0.8 \mathrm{~V}$ was applied to the glassy carbon with respect to Pt counter electrode. The dark particles, confirmed to be Pt by energydispersive X-ray spectroscopy (EDS), begin to appear when the voltage is applied and continue to grow with time. These in situ studies show the dynamics of electrodeposition from the micro- to the nanoscale under realistic growth conditions. In those in situ experiments, electrodeposition of Pt on glassy carbon cathode results in two-dimensional growth in the macroscopic regime. Highly dendritic - either dense branching or ramified islands - are formed at the solid-electrolyte interface which agrees well with the diffusion-limited-aggregation mode.

With this in situ TEM approach, we also demonstrate that the Mullins-Sekerka instability induced by initial surface roughness, combined with local enhancement of electric field, gives rise to non-uniform ramified deposition as a result of nucleation/growth at preferred locations. By creating local diffusion flow using an electron beam radiolysis effect, a large-scale propagation of the reaction front is also observed. Comparing the growth behavior under these different conditions provides new insight into the fundamental mechanisms of electrodeposition [3]. 


\section{References:}

[1] M J Williamson, RM Tromp, P M Vereecken, R. Hull, FM Ross, Nat. Mater. 2, (2003), P.532.

[2]. N de Jonge, FM Ross, Nat. Nanotechnol. 6, (2011), P.695.

[3] Use of the EM facilities in the Center for Nanoscale Materials, an Office of Science user facility, was supported by the U. S. Department of Energy, Office of Science, Office of Basic Energy Sciences, under Contract No. DE-AC02-06CH11357.
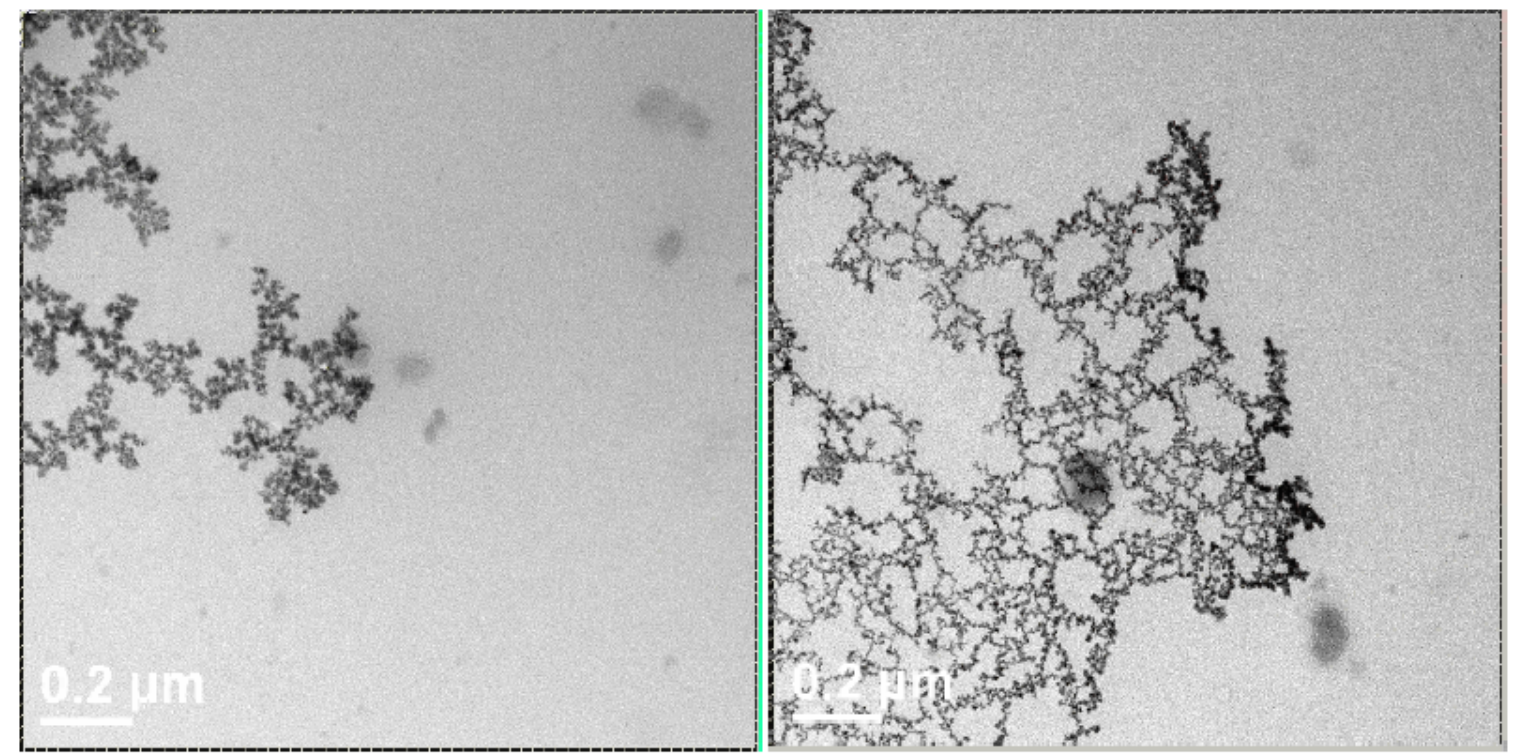

Figure 1. In situ records of Pt fractal growth during electrodeposition 